\title{
New scoring system for predicting percutaneous coronary intervention of chronic total occlusion success: Impact of operator's experience
}

\author{
Mohsen Mohandes ${ }^{1}$, Cristina Moreno ${ }^{1}$, Mónica Fuertes ${ }^{1}$, Sergio Rojas ${ }^{1}$, \\ Alberto Pernigotti ${ }^{1}$, Diego Zambrano, Victor Doblas ${ }^{1}$, \\ Francisco Fernández $z^{1}$, Jordi Guarinos ${ }^{1}$, Alfredo Bardají ${ }^{2}$ \\ ${ }^{1}$ Interventional Cardiology Unit, Cardiology Division, Joan XXIII University Hospital, \\ Pere Virgili Health Research Insitute (IISPV), Tarragona, Spain \\ ${ }^{2}$ Cardiology Division, Joan XXIII University Hospital, Pere Virgili Health Research Insitute (IISPV), \\ Tarragona, Spain
}

\begin{abstract}
Background: Several scoring systems have been developed in order to predict percutaneous coronary intervention (PCI) result of chronic total occlusion (CTO). The scores principally include anatomic and clinical variables. Operator experience is a decisive factor for achieving successful result. We sought to assess the real impact of operator growing experience on CTO-PCI success.

Methods: The angiographic and clinical variables of CTO-PCIs performed in our center between May 2007 and April 2021 were collected, and variables with potential association with procedural result were thoroughly reviewed. The influence of operator experience based on the number of previous CTO-PCIs was statistically assessed. A scoring system with combination of anatomic variables and operator experience was devised.

Results: A total of 540 PCIs in 457 patients were performed in our institution. The scoring model was developed from the derivation set (2/3 of the cohort). The final variables in logistic regression model were CTO length $\geq 20 \mathrm{~mm}$, blunt stump, vessel tortuosity $>45^{\circ}$ and operator experience $<100$ PCIs. The model showed good performance in the derivation set (area under curve [AUC]: 0.768; confidence interval [CI]: 0706-0.830; $p<0.001$ ) with no significant shrinkage in the validation set (AUC: 0.704; CI: 0.613-0.796; $p<0.001)$.

Conclusions: This new score (E-CTO score) adequately predict the probability of CTO-PCI failure. The model includes a variable representing operator experience along with other anatomic variables. (Cardiol J)
\end{abstract}

Key words: percutaneous coronary intervention, coronary occlusions, coronary arteries

\section{Introduction}

Although the proportion of patients undergoing percutaneous coronary intervention (PCI) of chronic total occlusion (CTO) has increased over the last decade [1], PCIs on these kinds of inter- vention continue to be challenging for interventionists, with lower procedural success rate and worse outcomes compared with other complex non-CTO-PCIs [2]. During the last decade several scoring systems have been devised to correlate angiographic and/or clinical variables with final PCI

Address for correspondence: Mohsen Mohandes, MD, Interventional Cardiology Unit, Cardiology Division, Joan XXIII University Hospital, Pere Virgili Health Research Institute (IISPV), Calle Dr Mallafré Guasch 4, 43007 Tarragona, Spain, tel: 0034-977295817, e-mail: mohandesmohsen@hotmail.com

Received: 7.07.2021

Accepted: 11.08 .2021

Early publication date: 23.09.2021

This article is available in open access under Creative Common Attribution-Non-Commercial-No Derivatives 4.0 International (CC BY-NC-ND 4.0) license, allowing to download articles and share them with others as long as they credit the authors and the publisher, but without permission to change them in any way or use them commercially. 
success [3-6] aimed to stratify procedure difficulty for operators dealing with a CTO. Although some anatomic features such as CTO length $\geq 20 \mathrm{~mm}$, blunt stump and calcification, are commonly related to the procedural success in different scoring system [3, 6], CTO-PCI is highly operator dependent in such a way that more seasoned operators can achieve a high level of success in more complex cases [7].

Operator expertise is something that has been presupposed in different scoring systems but its real impact as an independent factor for CTO-PCI success has not yet been adequately scrutinized and the information in this regard is scarce in literature. We sought to assess the predictive variables for CTO-PCI success in our entire cohort since the installation of the program in our institution, including the contribution of operator experience as a potential independent factor.

\section{Methods}

The CTO program was established in our institution in May 2007, with a lower volume of interventions during its initial steps and at least 50 procedures a year since 2013 . Five workshops with Japanese experts in CTO-PCI over 5 consecutive years were carried out in our hospital, aiming to improve the experience of local operators. A CTO was defined as the presence of a coronary artery obstruction with absent antegrade flow through the lesion (thrombolysis in myocardial infarction [TIMI]) grade 0 flow standing for more than 3 months [8]. All data related to the procedures including those angiographic variables with potential influence on the procedural result were prospectively introduced in our database. The angiographic variables were re-examined again by 2 members of our cath lab. In case of discrepancy the opinion of a third examiner was requested and a final consensus was established.

A blunt stump was defined as absence of tapered tip or dimple at the entry point of the CTO, indicating the direction of the true lumen. Calcification was considered if any evident calcification was detected in CTO segment regardless of its severity. The attribution of tortuosity was based on the presence of bending $>45^{\circ}$ within CTO segment. CTO length was measured in therapeutic procedure while collaterals supplied the CTO distal segment which is more representative of true distance of the occlusion [9].

Upon completing the review, 50 randomly selected cases were inspected and the level of concordance between 2 observers was estimated so as to assess and resolve any possible interobserver bias.

Double access was employed in case of contralateral collateral supply to the CTO, with radial approach for the contralateral injection used in the majority of cases. The antegrade approach was the preferred strategy especially during the initial steps of our experience and retrograde access was incorporated as the level of expertise increased in our center. Retrograde approach was defined when an attempt was made to cross collateral channels supplying the distal part of the occluded vessel retrogradely [10]. Technical success was defined as achievement of TIMI grade 2 or greater antegrade flow in all $\geq 2.5 \mathrm{~mm}$ distal branches with $<30 \%$ residual stenosis of the target CTO lesion alt procedure end [8].

With the exception of few cases, the vast majority of CTO-PCIs were performed by a single dedicated operator.

Complications such as in-hospital death, periprocedural myocardial infarction (MI), coronary perforation requiring pericardiocentesis, major vascular complications needing percutaneous or surgical intervention and stroke were reported. Peri-procedural MI assignment was in accordance with the universal definition of PCI-related MI [11]. All patients signed informed consent before undergoing the procedure.

\section{Statistical analysis}

Continuous variables were presented as mean \pm standard deviation and categorical variables were expressed as absolute numbers and percentages. The Student $t$ statistic was used to compare quantitative variables between two groups and the $\chi^{2}$ or Fisher exact test were applied as appropriate to evaluate the association between qualitative variables. Based on the gradual improvement in the procedural success over time especially after first block of 100 cases, a binary variable of PCIs number more or less than 100 was created in order to assess the influence of accumulative experience on the final success. The entire cohort was randomly divided into derivation and validation sets at a ratio of 2:1. Odds ratios (ORs) of angiographic and clinical variables with a plausible relation to procedural failure such as CTO stump, bending, CTO length, calcification, previous failed attempt, ostial location, target vessel, distal vessel visibility, vessel diameter $<2.5 \mathrm{~mm}$, age, sex, previous $\mathrm{MI}$, previous coronary artery bypass grafting (CABG) and multivessel disease [3, 4, 12-14], were individually assessed and those with a $\mathrm{p}<0.10$ were 
selected for introducing in the multivariate model. Even if the $p$ value for candidate variables was not statistically significant but had an OR greater than or equal to 1.2 , the variable was kept in the model due to its potential plausible contribution to the model performance. A predictive model with a combination of variables associated with procedural failure with multivariate logistic regression was constructed using both forward and backward stepwise methods in the derivation model. A scoring model was developed in the derivation set giving 1 point to each variable remaining in the multivariate logistic regression model according to their magnitude of beta coefficient. The performance of the model was assessed by the receiver operator characteristic (ROC) curve. The discriminatory performance of the model was validated by comparing the ROC curve of the derivation set with that of the validation set. A linear trend in categorical variables was evaluated by the polynomial test in the logistic regression model.

The goodness-of-fit of the model was estimated using the Hosmer and Lemeshow test in order to evaluate any possible discrepancy between observed and expected values.

Finally, a prognostic index statistic model was developed with different combinations of predictive variables so as to assess the relative risk of procedural failure in fictitious individuals. Statistical package of SPSS 19 was used for all analysis and a $p$ value of $<0.05$ was considered to indicate statistical significance.

\section{Results}

A total of 540 PCIs in 457 patients between May 2007 and April 2021 were performed at the cited institution. The average age of the entire cohort was $65 \pm 10$ years old and $246(45.6 \%)$ patients had diabetes. In 1 patient, there were missing values with regard to some angiographic variables related to procedural success. The level of interobserver concordance (kappa index) in 50 randomly selected cases for the estimation of variables associated with the procedural result was 0.8 .

Out of 492 first-attempt CTO-PCIs, 394 $(80.1 \%)$ were successful. A total of 48 new-attempts on previously failed procedures were carried out of which 36 (75\%) were successful. The overall procedural success by patient and artery was $86 \%$ in the entire cohort (Fig. 1). The success rate improved over time especially after the first block of 100 cases with a significant $\mathrm{p}$ for linear trend ( $<<0.001$; Fig. 2).

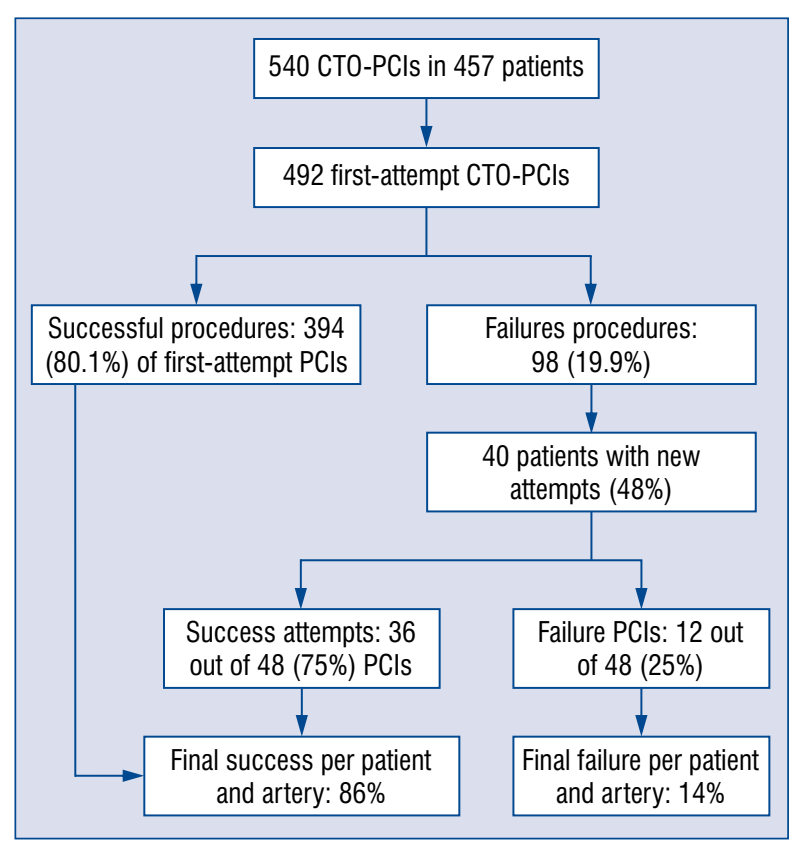

Figure 1. Flowchart of overall procedural success including reattempted interventions in the entire cohort; CTO - chronic total occlusion; $\mathrm{PCl}$ - percutaneous coronary intervention.

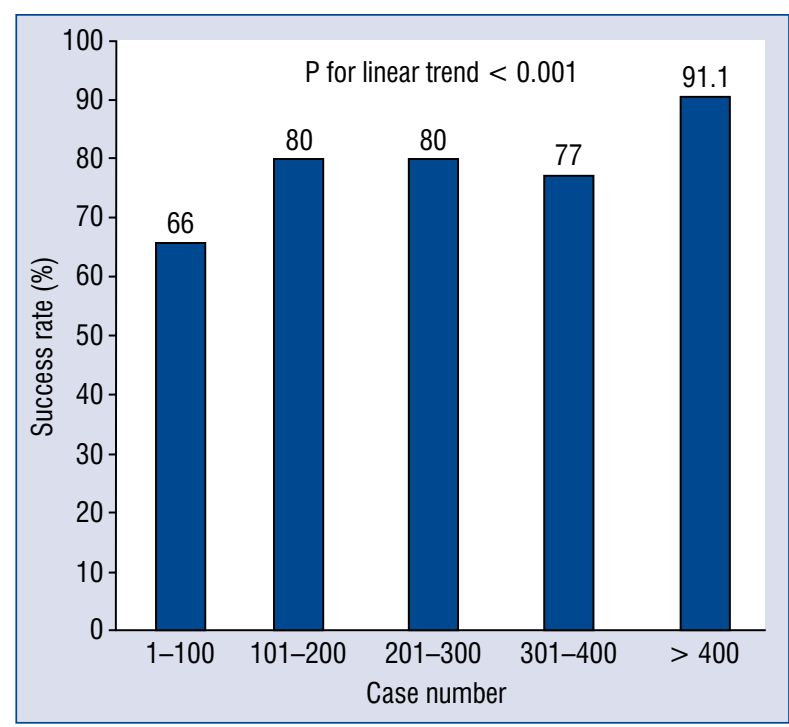

Figure 2. Procedural success rate according to growing case performance in the entire cohort.

Comparison of baseline, angiographic characteristics and procedural outcomes in failed and successful groups in the derivation set is depicted in Table 1 . The failed group was represented by significantly more complex lesions according to J-CTO score $(2 \pm 1.1$ vs. $1.1 \pm 1$; p < 0.001) with regard to the successful group. Both procedural and 
Table 1. Comparison of baseline, angiographic characteristics and outcomes between successful and failed procedures in derivation group.

\begin{tabular}{|c|c|c|c|}
\hline & Successful procedure $(n=285)$ & Failed procedure $(n=76)$ & $\mathbf{P}$ \\
\hline Age [years] & $64.6 \pm 11.1$ & $63.9 \pm 10.6$ & 0.65 \\
\hline Female & $47(16.5 \%)$ & $7(9.2 \%)$ & 0.11 \\
\hline BMI $\left[\mathrm{kg} / \mathrm{m}^{2}\right]$ & $28.6 \pm 4.6$ & $28.6 \pm 4.2$ & 0.9 \\
\hline Hypertension & $210(73.7 \%)$ & $59(77.6 \%)$ & 0.48 \\
\hline Diabetes & $128(44.9 \%)$ & $37(48.7 \%)$ & 0.56 \\
\hline Dyslipidemia & $192(67.4 \%)$ & $60(78.9 \%)$ & 0.051 \\
\hline Smoking & $167(58.6 \%)$ & $48(63.2 \%)$ & 0.47 \\
\hline Previous MI & $123(43.2 \%)$ & $37(48.7 \%)$ & 0.39 \\
\hline Previous CABG & $23(8.1 \%)$ & $7(9.2 \%)$ & 0.75 \\
\hline MD & $180(63.2 \%)$ & $49(64.5 \%)$ & 0.83 \\
\hline Target vessel: & & & 0.33 \\
\hline LAD & $150(34.9 \%)$ & $28(25.5 \%)$ & \\
\hline LCX & $80(18.6 \%)$ & $19(17.3 \%)$ & \\
\hline $\mathrm{RCA}$ & $187(43.5 \%)$ & $62(56.4 \%)$ & \\
\hline DG & $6(1.4 \%)$ & $1(0.9 \%)$ & \\
\hline LM & $2(0.5 \%)$ & $0(0 \%)$ & \\
\hline $\mathrm{RI}$ & $3(0.7 \%)$ & $0(0 \%)$ & \\
\hline SVG & & $2(0.5 \%)$ & $0(0 \%)$ \\
\hline Ejection farction $<40 \%$ & $45(15.8 \%)$ & $11(14.5 \%)$ & 0.78 \\
\hline J-CTO score & $1.1 \pm 1$ & $2 \pm 1.1$ & $<0.001$ \\
\hline E-CTO score & $0.77 \pm 0.88$ & $1.76 \pm 1$ & $<0.001$ \\
\hline Easy & $135(47.5 \%)$ & $10(13.2 \%)$ & \\
\hline Intermediate & $94(33.1 \%)$ & $18(23.7 \%)$ & \\
\hline Difficult & $40(14.1 \%)$ & $29(38.2 \%)$ & \\
\hline Very difficult & $15(5.3 \%)$ & $19(25 \%)$ & \\
\hline Retrograde* & $31(10.8 \%)$ & $24(31.6 \%)$ & $<0.001$ \\
\hline Procedure time [min] & $145 \pm 73$ & $200 \pm 80$ & $<0.001$ \\
\hline Fluoroscopy time [min] & $64 \pm 42$ & $103 \pm 42$ & $<0.001$ \\
\hline Contrast medium & $251 \pm 99$ & $271 \pm 98$ & 0.13 \\
\hline In-hospital death & $2(0.7 \%)$ & $1(1.3 \%)$ & 0.51 \\
\hline Perforation & $0(0 \%)$ & $2(2.6 \%)$ & 0.04 \\
\hline MV complication & $3(1.1 \%)$ & $0(0 \%)$ & 1 \\
\hline $\mathrm{MI}$ & $2(0.7 \%)$ & $6(7.9 \%)$ & $<0.001$ \\
\hline
\end{tabular}

*The retrograde approach in the entire cohort was $15.4 \%$. BMI — body mass index; CABG — coronary artery bypass graft; DG — diagonal artery; E-CTO - operator experience-chronic total occlusion; LAD — left anterior descending coronary artery; LCX - left circumflex; LM - left main; $\mathrm{MI}$ - myocardial infarction; MD - multivessel disease; MV complication - major vascular complication; RCA — right coronary artery; $\mathrm{RI}$ - ramus intermedius; SVG — saphenous vein graft

fluoroscopy time were significantly longer in the failed group compared with those of the successful group (200 \pm 80 vs. $145 \pm 73 \mathrm{~min}$ and $103 \pm 42$ vs. $64 \pm 42 \mathrm{~min}$, respectively).

The comparison of baseline, clinical and angiographic characteristics as well as procedural outcomes between derivation and validation sets revealed no significant between-group-difference (Table 2). The right coronary artery was the most frequently targeted artery representing 166 (46\%) and $83(46.4 \%)$ cases in derivation and validation groups, respectively.

Those variables potentially related with technical success are depicted in Table 3. Those variables with $\mathrm{p}<0.10$ and/or $\mathrm{OR} \geq 1.2$ were selected for the subsequent analysis in the multivariate model.

In the logistic regression model the final variables using both forward and backward stepwise 
Table 2. Baseline patient characteristics, angiographic characteristics and procedural outcomes comparison between derivation and validation groups.

\begin{tabular}{|c|c|c|c|}
\hline & Derivation set $(n=361)$ & Validation set $(n=179)$ & $\mathbf{P}$ \\
\hline Age [years] & $64.5 \pm 11$ & $66.4 \pm 10.6$ & 0.053 \\
\hline Female & $54(15 \%)$ & $30(16.8 \%)$ & 0.6 \\
\hline BMI $\left[\mathrm{kg} / \mathrm{m}^{2}\right]$ & $28.6 \pm 4.5$ & $29.11 \pm 4.9$ & 0.25 \\
\hline Hypertension & $92(25.5 \%)$ & $44(24.6 \%)$ & 0.82 \\
\hline Diabetes & $196(54.3 \%)$ & $98(54.7 \%)$ & 0.92 \\
\hline Dyslipidemia & $109(30.2 \%)$ & $49(27.4 \%)$ & 0.5 \\
\hline Smoking & $215(59.6 \%)$ & $107(59.8 \%)$ & 0.96 \\
\hline Previous MI & $160(44.3 \%)$ & $85(47.5 \%)$ & 0.49 \\
\hline Previous CABG & $30(8.3 \%)$ & $13(7.3 \%)$ & 0.67 \\
\hline MD & $229(63.4 \%)$ & $114(63.7 \%)$ & 0.95 \\
\hline Target vessel: & & & 0.94 \\
\hline LAD & $119(33 \%)$ & $59(33 \%)$ & \\
\hline LCX & $65(18 \%)$ & $34(19 \%)$ & \\
\hline RCA & $166(46 \%)$ & $83(46.4 \%)$ & \\
\hline DG & $5(1.4 \%)$ & $2(1.1 \%)$ & \\
\hline LM & $2(0.6 \%)$ & $0(0 \%)$ & \\
\hline $\mathrm{RI}$ & $3(0.8 \%)$ & $0(0 \%)$ & \\
\hline SVG & $1(0.3 \%)$ & $1(0.6 \%)$ & \\
\hline Ejection fraction $<40 \%$ & $56(15.5 \%)$ & $24(13.4 \%)$ & 0.52 \\
\hline J-CTO score & $1.31 \pm 1.11$ & $1.33 \pm 1.14$ & 0.84 \\
\hline E-CTO score & $1 \pm 0.99$ & $1 \pm 0.96$ & 0.83 \\
\hline Retrograde & $54(15.2 \%)$ & $28(15.6 \%)$ & 0.44 \\
\hline Procedure time [min] & $157 \pm 78$ & $151 \pm 67$ & 0.4 \\
\hline Fluoroscopy time [min] & $71 \pm 45$ & $71 \pm 44$ & 0.97 \\
\hline Contrast medium & $256 \pm 99$ & $249 \pm 76$ & 0.43 \\
\hline Procedural success & $285(78.9 \%)$ & $145(81 \%)$ & 0.57 \\
\hline In-hospital death & $3(0.8 \%)$ & $0(0 \%)$ & 0.55 \\
\hline Perforation & $2(0.6 \%)$ & $1(2.6 \%)$ & 1 \\
\hline MV complication & $3(0.8 \%)$ & $0(0 \%)$ & 0.55 \\
\hline MI & $8(2.2 \%)$ & $4(2.2 \%)$ & 1 \\
\hline
\end{tabular}

$\mathrm{BMI}$ - body mass index; CABG — coronary artery bypass graft; DG — diagonal artery; E-CTO - operator experience-chronic total occlusion; LAD — left anterior descending coronary artery; LCX - left circumflex; LM — left main; MI — myocardial infarction; MD — multivessel disease; MV complication — major vascular complication; RCA — right coronary artery; RI — ramus intermedius; SVG — saphenous vein graft

methods were CTO length $\geq 20 \mathrm{~mm}$, blunt stump, vessel tortuosity $>45^{\circ}$ and accumulative experience less than 100 CTO-PCIs (Table 4). E-CTO score was derived from variables remaining in the multivariate model. As the magnitude of beta coefficient was close to unit, 1 point was given to each variable. The score classified the CTO as easy ( 0 point), intermediate ( 1 point), difficult ( 2 points) and very difficult ( $\geq 3$ points).

Receiver operator characteristic analysis of the model showed good performance in derivation set (area under curve [AUC]: 0.768; CI: 0706-0.830; $p<0.001)$ with no significant shrink- age in validation set (AUC: 0.704; CI: 0.613-0.796; $\mathrm{p}<0.001$ ). Additional assessment of AUC represented in ROC curve for variables included in J-CTO model was performed with AUC: 0.747 (CI: $0.68-0.81 ; \mathrm{p}<0.001$ ) for derivation and AUC: 0.617 (CI: $0.51-0.72 ; \mathrm{p}=0.034$ ) for validation set, respectively (Fig. 3).

The model showed good calibration both in derivation and validation groups according to the Hosmer and Lemeshow test (X2: 1.84 ; p: 0.87 for derivation and X2: 9.23 ; p: 0.16 in validation group, respectively).

According to the present study scores the probability of PCI failure increased from class 0 to 
Table 3. Preselection of variables in univariate model as a previous step for the construction of the multivariate model. Variables with $p<0.10$ and/or odds ratio $\geq 1.2$ were kept in the model for the subsequent multivariate statistical analysis.

\begin{tabular}{lccc}
\hline Variables & Odds ratio $(95 \% \mathrm{Cl})$ & Beta coefficient & $\mathbf{P}$ \\
\hline Length & $3.07(1.79-5.26)$ & 1.12 & $<0.001$ \\
Stump & $3.9(2.35-6.76)$ & 1.38 & $<0.001$ \\
Tortuosity & $4.2(2.42-7.25)$ & 1.43 & $<0.001$ \\
CN & $2.4(1.31-4.41)$ & 0.88 & 0.004 \\
Calcification & $1.4(0.82-2.33)$ & 0.34 & 0.19 \\
Sex & $1.95(0.84-4.5)$ & 0.67 & 0.12 \\
Previous MI & $1.25(0.75-2.07)$ & 0.22 & 0.39 \\
Ostial location & $1.22(0.53-2.83)$ & 0.20 & 0.64 \\
Previous attempt & $1.34(0.55-3.30)$ & 0.29 & 0.52 \\
Visibility & $1.74(0.73-4.18)$ & 0.56 & 0.21 \\
Diameter $<2.5 \mathrm{~mm}$ & $1.25(0.72-2.16)$ & 0.22 & 0.43 \\
Age & $0.99(0.97-1.01)$ & -0.005 & 0.65 \\
CABG & $1.15(0.48-2.80)$ & 0.14 & 0.75 \\
MD & $1.06(0.62-1.8)$ & 0.057 & 0.83 \\
Artery & $1.07(0.85-1.36)$ & 0.071 & 0.56 \\
\hline
\end{tabular}

CABG — coronary artery bypass graft; $\mathrm{Cl}$ — confidence interval; $\mathrm{CN}$ - case number; $\mathrm{MD}$ - multivessel disease; $\mathrm{MI}$ - myocardial infarction

Table 4. Variables associated with CTO-PCI failure in multivariate logistic regression model derived from derivation subset. Four independent predictors were identified by the forward and backward method: chronic total occlusion length $\geq 20 \mathrm{~mm}$, blunt stump, bending $>45^{\circ}$ and accumulative experience $<100$ cases $(n=360)$. E-CTO score was derived from variables remaining in the multivariate model giving one point to each variable as the magnitude of beta coefficients was close to unit.

\begin{tabular}{lcccc}
\hline Predictors & Odds ratio $(95 \% \mathrm{Cl})$ & Beta coefficient & $P$ & E-CTO score \\
\hline Length & $2.06(1.13-3.7)$ & 0.723 & 0.018 & 1 \\
Stump & $3.69(2.0-6.69)$ & 1.3 & $<0.001$ & 1 \\
Tortuosity & $2.67(1.47-4.8)$ & 0.98 & 0.001 & 1 \\
CN & $3.20(1.61-6.3)$ & 1.16 & 0.001 & 1 \\
\hline
\end{tabular}

$\mathrm{Cl}$ - confidence interval; $\mathrm{CN}$ - case number; E-CTO score classification: $0=$ easy; $1=$ intermediate; $2=$ difficult; $\geq 3=$ very difficult

3 (easy to very difficult CTO) with significant $\mathrm{p}$ for linear trend both in derivation and validation sets (p < 0.001; Fig. 4).

Prognostic index derived from the combination of different values of 4 variables in fictitious individuals predicts a relative risk (RR) of 1 for the case with 0 points for all 4 independent variables (the easiest case). If a fictitious case has 1 point in all 4 variables, the RR of failure is 11.4 times more with respect to the easiest case (Table 5).

\section{Discussion}

In this single center experience in CTO-PCIs the independent predictors associated with the procedural failure were CTO blunt stump, the occlusion length $\geq 20 \mathrm{~mm}$, tortuosity $>45^{\circ}$ in addition to the operator's accumulative experience. The study reveals operator expertise, defined as less than 100 previous CTO-PCIs performance, as an independent predictor for the procedural failure.

The construction of scoring systems in CTO-PCI are aimed to predict and correlate the level of procedural difficulty with final procedural result. Scoring systems provide interventionists with a quantitative measure of procedural difficulty and the final success rate, which is beneficial when planning a PCI. Moreover, the CTO scoring models enable us to tailor the case difficulty to the operator level of experience so that more complex cases 

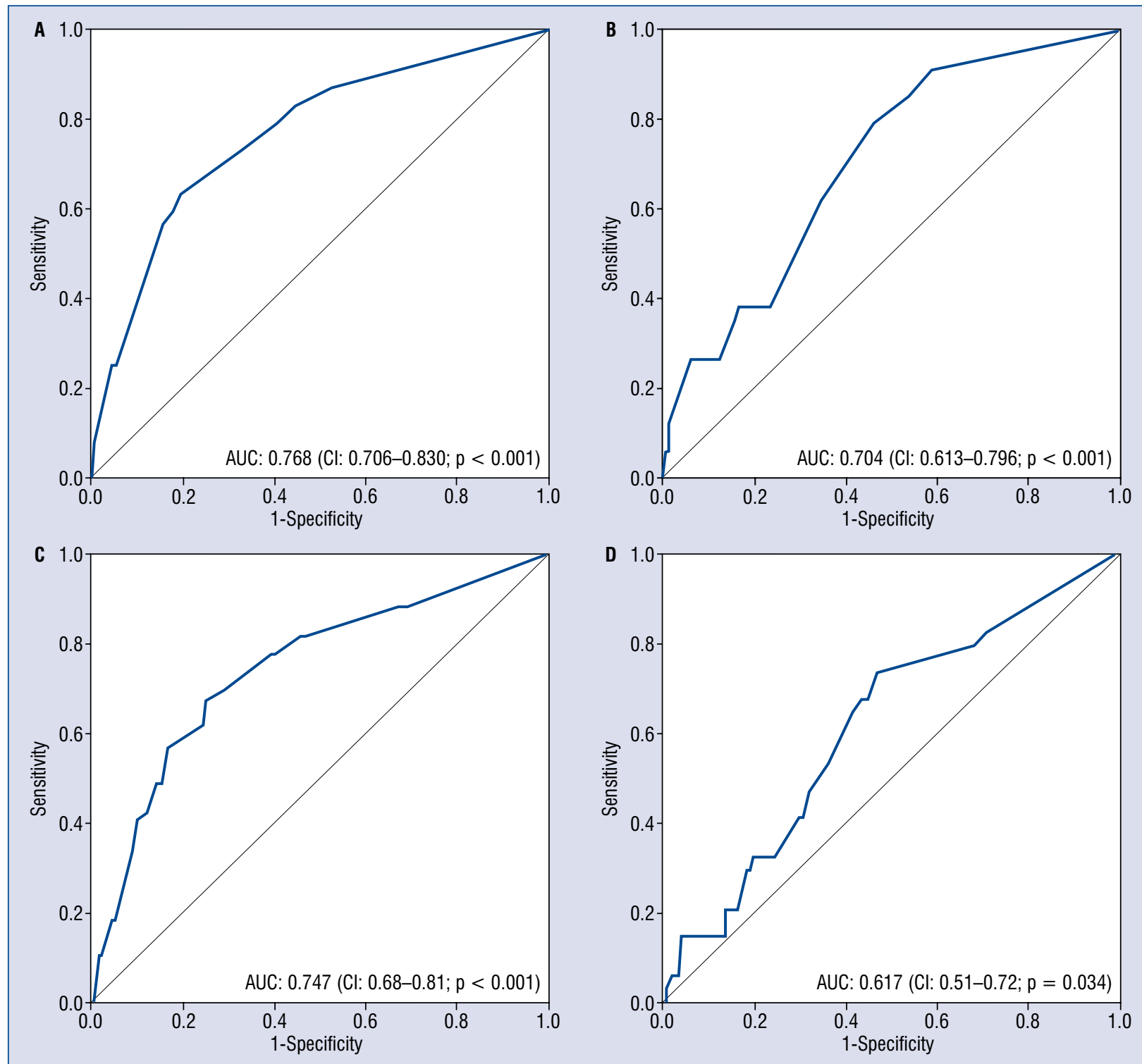

Figure 3. Receiver operator characteristic $(R O C)$ curve in derivation $(A)$ and validation $(B)$ sets based on variables included in E-CTO (CTO length, stump, bending and operator experience) and ROC curve in derivation (C) and validation (D) sets based on variables included in J-CTO score (CTO length, stump, bending, calcification and previous failed attempt); CTO - chronic total occlusion; AUC - area under curve; $\mathrm{Cl}$ - confidence interval.

could be performed with more seasoned operators [15]. Many scoring models mainly encompass anatomic variables such as calcification, ambiguous stump, CTO length and bending. Other scores found the association between CTO-PCI failure and several clinical variables including previous MI, previous $\mathrm{CABG}$ and age in addition to anatomic variables $[12,13]$.

What is commonly assumed in different scoring systems, is the fact that the submitted cases were performed by expert operators as it is specifically mentioned in the Euro-CTO castle score [13] which analyzed the outcome of 20,000 CTO-PCIs in centers with more than 50 cases a year.

As mentioned earlier, CTO-PCIs are highly operator-dependent. According to the Euro-CTO club registry, operators who have performed more than $300 \mathrm{CTO}-\mathrm{PCI}$ and maintain an annual procedure number of at least 50 cases can achieve a success rate of more than $85 \%$ [16].

Zein et al. [17] analyzed the association of operator experience in CTO-PCI success according to previous case number performance at the time of CTO-PCI $(<12$, between 12 and 33, and 


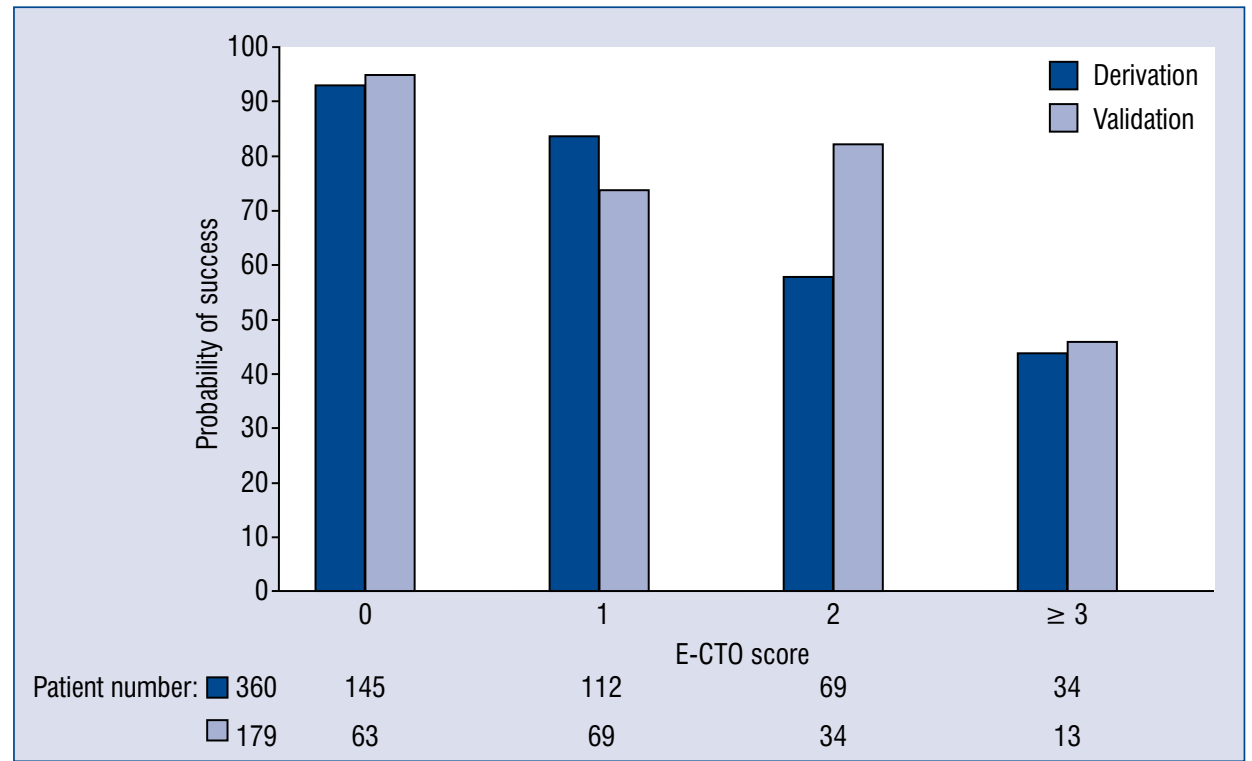

Figure 4. Probability of percutaneous coronary intervention success according to the magnitude of E-CTO score in derivation and validation set.

Table 5. Prognostic index: elaboration of fictitious subjects with different combinations of unfavorable predictors for CTO-PCI success and their relative risk (RR) for procedural failure.

\begin{tabular}{lccccc}
\hline Length & Stump & Bending & CN & Prop & RR \\
\hline 0 & 0 & 0 & 0 & $\mathbf{0 . 0 6 9 0}$ & $\mathbf{0 . 9 9 9 3 ^ { * }}$ \\
0 & 0 & 1 & 0 & 0.1097 & 1.5892 \\
1 & 0 & 0 & 0 & 0.1354 & 1.9617 \\
0 & 0 & 0 & 1 & 0.2025 & 2.9343 \\
1 & 0 & 1 & 0 & 0.2066 & 2.9937 \\
0 & 1 & 0 & 0 & 0.2332 & 3.3796 \\
0 & 0 & 1 & 1 & 0.2969 & 4.3024 \\
0 & 1 & 1 & 0 & 0.3359 & 4.8678 \\
1 & 0 & 0 & 1 & 0.3492 & 5.0613 \\
1 & 1 & 0 & 0 & 0.3913 & 5.6709 \\
1 & 0 & 1 & 1 & 0.4716 & 6.8346 \\
0 & 1 & 0 & 1 & 0.5104 & 7.3970 \\
1 & 1 & 1 & 0 & 0.5167 & 7.4882 \\
0 & 1 & 1 & 1 & 0.6342 & 9.1912 \\
1 & 1 & 0 & 1 & 0.6878 & 9.9688 \\
1 & 1 & 1 & 1 & 0.7856 & 11.3858 \\
\hline
\end{tabular}

*Reference value. According to this index if a patient has none of the unfavorable predictors the relative risk of procedural failure is almost 1 (the easiest and the reference case) and if all four unpropitious predictors are present the relative risk increases by 11.4 times (the most unfavorable case) with regard to the referent case.

$\mathrm{CN}-$ case number $(<100 \mathrm{PCls}=1)$; Prop - the failure probability

234). CTO-PCI success rate increased significantly throughout these 3 tertiles $(44.9 \%, 54.5 \%$ and $64.5 \%$ ) respectively according to this study.
Japanese CTO-PCI expert registry reported results of CTO-PCIs performed by 41 highly experienced operators [18] with a high technical success (89.9\%) and a frequent selection of the retrograde approach (in $27.8 \%$ of cases) as the first strategy. The study indicates that highly seasoned operators are more familiar with more complex techniques such as the retrograde approach, enabling them to achieve a high technical success rate. It is worth mentioning that the retrograde approach in the current entire cohort was lower than that reported in the Japanese study and accounted for $15.4 \%$ of cases which reveals the fact that more complex techniques were incorporated gradually as experience increased over time.

Habara et al. [19] compared the CTO-PCIs success rate in high-volume centers ( $>50$ CTO-PCIs per year) with that of low-volume centers ( $<50$ CTO-PCIs per year) in Japan and found a statistically significant between-group difference ( $90.6 \%$ vs. $85.6 \%$, respectively). They concluded that operator experience was a key factor for this disparity.

Therefore, the impact of operator experience on CTO-PCI technical success is something that is commonly accepted but its influence and impact on a scoring system not have been previously addressed to date. Based on this rationale and in agreement with our progressive improvement in CTO-PCIs it was considered plausible to include the operator expertise along with angiographic 
variables in a scoring model. Although adjusted to the reality of our center in terms of logistic and technical strategies, the scoring model could acceptably predict the probability of PCI failure in the cohort. Results were consistent with the evidence in literature regarding a clear impact of operator experience on CTO-PCI outcomes, which we expressed and confirmed in a scoring model with a statistical base.

As previously reported the J-CTO score fitness for technical success prediction in the present cohort, as characterized by growing experience over time it was concluded that the score showed better performance as the level of experience increased [20]. Indeed, the inclusion of operator experience as an independent predictor in a new devised scoring system (E-CTO score) improved the performance of the model comparing with J-CTO score as depicted in ROC curves earlier (Fig. 3). The scoring model predicts acceptably the procedural result and the elaboration of prognostic index provides additional information about the relative risk of PCI failure in each case based on the combination of values of different variables. This information can be shared with the patient and referring physician before proceeding with the intervention.

\section{Limitations of the study}

There are several limitations in this study. Firstly, this is a single center study with cases performed mainly by a single operator and although the study has internal validation, its results cannot be extrapolated to other centers. Therefore, the study needs external validation in centers with similar logistic and technical characteristics in terms of CTO-PCIs. Secondly, the incorporation of more complex techniques such as the retrograde approach was gradual in our institution and not in the first step of our CTO-PCI program. Increased application of retrograde approach might have changed the results. As mentioned previously, in the Japanese series retrograde approach was used more frequently than in the present cohort. Thirdly, improvement and incorporation of new devices for CTO-PCI over time probably is another contributing factor for procedural success, and was not scrutinized in the current study. Finally, the use of antegrade dissection reentry with dedicated devices such as the CrossBoss catheter, Stingray balloon and wires (Boston scientific, Natick, Massachusets) that could have made the procedure faster [21,22] was limited by operator experience in very few cases. Consequently, the results cannot be extrapolated from the present center to centers in which this technique is more frequently used.

\section{Conclusions}

This new scoring system called E-CTO score comprising anatomic variables such as blunt stump, tortuosity $>45^{\circ}$, CTO length $\geq 20 \mathrm{~mm}$, along with operator previous experience $<100$ CTO-PCIs shows a good prediction capacity for technical success with an AUC $>0.7$. The study has for the first time included operator experience in a scoring model, which can serve as a guide for centers with a dedicated CTO program with growing experience in this field.

\section{Conflict of interest: None declared}

\section{References}

1. Brilakis E, Banerjee S, Karmpaliotis D, et al. Procedural outcomes of chronic total occlusion percutaneous coronary intervention. JACC: Cardiovascular Interventions. 2015; 8(2): 245-253, doi: 10.1016/j.jcin.2014.08.014.

2. Azzalini L, Carlino M, Bellini B, et al. Long-Term outcomes of chronic total occlusion recanalization versus percutaneous coronary intervention for complex non-occlusive coronary artery disease. Am J Cardiol. 2020; 125(2): 182-188, doi: 10.1016/j. amjcard.2019.10.034, indexed in Pubmed: 31759516.

3. Morino Y, Abe M, Morimoto T, et al. Predicting successful guidewire crossing through chronic total occlusion of native coronary lesions within 30 minutes. JACC: Cardiovascular Interventions. 2011; 4(2): 213-221, doi: 10.1016/j.jcin.2010.09.024.

4. Christopoulos G, Kandzari DE, Yeh RW, et al. Development and Validation of a Novel Scoring System for Predicting Technical Success of Chronic Total Occlusion Percutaneous Coronary Interventions: The PROGRESS CTO (Prospective Global Registry for the Study of Chronic Total Occlusion Intervention) Score. JACC Cardiovasc Interv. 2016; 9(1): 1-9, doi: 10.1016/j. jcin.2015.09.022, indexed in Pubmed: 26762904.

5. De Jin C, Kim MH, Kim SJ, et al. Predicting successful recanalization in patients with native coronary chronic total occlusion: the busan CTO score. Cardiology. 2017; 137(2): 83-91, doi: 10.1159/000455824, indexed in Pubmed: 28171874.

6. Guelker JE, Bansemir L, Ott R, et al. Validity of the J-CTO Score and the CL-Score for predicting successful CTO recanalization. Int J Cardiol. 2017; 230: 228-231, doi: 10.1016/j.ijcard.2016.12.165, indexed in Pubmed: 28041697.

7. Christopoulos G, Wyman RM, Alaswad K, et al. Clinical utility of the japan-chronic total occlusion score in coronary chronic total occlusion interventions: results from a multicenter registry. Circ Cardiovasc Interv. 2015; 8(7): e002171, doi: 10.1161/CIRCINTERVENTIONS.114.002171, indexed in Pubmed: 26162857.

8. Ybarra LF, Rinfret S, Brilakis ES, et al. Definitions and clinical trial design principles for coronary artery chronic total occlusion therapies: CTO-ARC consensus recommendations. Circu- 


\section{Cardiology Journal}

lation. 2021; 143(5): 479-500, doi: 10.1161/CIRCULATIONAHA.120.046754, indexed in Pubmed: 33523728.

9. Morino Y, Abe M, Morimoto T. Predicting Successful Guidewire Crossing Through Chronic Total Occlusion of Native Coronary Lesions Within 30 Minutes The J-CTO (Multicenter CTO Registry in Japan) Score as a Difficulty Grading and Time Assessment Tool. JACC Cardiovasc Interv. 2011; 4(2): 213-221, doi: 10.1016/j.jcin.2010.09.024, indexed in Pubmed: 21349461.

10. Sumitsuji S, Inoue K, Ochiai M, et al. Fundamental wire technique and current standard strategy of percutaneous intervention for chronic total occlusion with histopathological insights. JACC Cardiovasc Interv. 2011; 4(9): 941-951, doi: 10.1016/j. jcin.2011.06.011, indexed in Pubmed: 21939933.

11. Thygesen K, Alpert J, Jaffe A, et al. Fourth universal definition of myocardial infarction (2018). Eur Heart J. 2018; 40(3): 237-269, doi: 10.1093/eurheartj/ehy462.

12. Alessandrino $\mathrm{G}$, Chevalier $\mathrm{B}$, Lefèvre $\mathrm{T}$, et al. A clinical and angiographic scoring system to predict the probability of successful first-attempt percutaneous coronary intervention in patients with total chronic coronary occlusion. JACC Cardiovasc Interv. 2015; 8(12): 1540-1548, doi: 10.1016/j.jcin.2015.07.009, indexed in Pubmed: 26493246.

13. Szijgyarto $Z$, Rampat R, Werner GS, et al. Derivation and Validation of a Chronic Total Coronary Occlusion Intervention Procedural Success Score From the 20,000-Patient EuroCTO Registry: The EuroCTO (CASTLE) Score. JACC Cardiovasc Interv. 2019; 12(4): 335-342, doi: 10.1016/j.jcin.2018.11.020, indexed in Pubmed: 30711551.

14. Mohandes M, Guarinos J, Rodríguez J, et al. [Negative angiographic markers in percutaneous coronary intervention of chronic total occlusions]. Arch Cardiol Mex. 2018; 88(2): 93-99, doi: 10.1016/j.acmx.2017.01.008, indexed in Pubmed: 28268135.

15. Harding SA, Wu EB, Lo S, et al. A new algorithm for crossing chronic total occlusions from the asia pacific chronic total occlusion club. JACC Cardiovasc Interv. 2017; 10(21): 2135-2143, doi: 10.1016/j.jcin.2017.06.071, indexed in Pubmed: 29122129.

16. Galassi AR, Werner GS, Boukhris M, et al. Percutaneous recanalisation of chronic total occlusions: 2019 consensus document from the EuroCTO Club. EuroIntervention. 2019; 15(2): 198-208, doi: 10.4244/EIJ-D-18-00826, indexed in Pubmed: 30636678.

17. Zein R, Seth M, Othman H, et al. Association of operator and hospital experience with procedural success rates and outcomes in patients undergoing percutaneous coronary interventions for chronic total occlusions: insights from the blue cross blue shield of Michigan cardiovascular consortium. Circ Cardiovasc Interv. 2020; 13(8): e008863, doi: 10.1161/CIRCINTERVENTIONS.119.008863, indexed in Pubmed: 32791954.

18. Suzuki Y, Tsuchikane E, Katoh O, et al. Outcomes of percutaneous coronary interventions for chronic total occlusion performed by highly experienced japanese specialists: the first report from the japanese CTO-PCI expert registry. JACC Cardiovasc Interv. 2017; 10(21): 2144-2154, doi: 10.1016/j.jcin.2017.06.024, indexed in Pubmed: 29055764.

19. Habara M, Tsuchikane E, Muramatsu T, et al. Comparison of percutaneous coronary intervention for chronic total occlusion outcome according to operator experience from the Japanese retrograde summit registry. Catheter Cardiovasc Interv. 2016; 87(6): 1027-1035, doi: 10.1002/ccd.26354, indexed in Pubmed: 26719060.

20. Mohandes M, Moreno C, Rojas S, et al. J-chronic total occlusion score predictive capacity for percutaneous coronary intervention success of chronic total occlusion: Results from a European single center cohort with progressive experience over time. Cardiol J. 2021 [Epub ahead of print], doi: 10.5603/CJ.a2021.0058, indexed in Pubmed: 34125431.

21. Whitlow PL, Burke MN, Lombardi WL, et al. Use of a novel crossing and re-entry system in coronary chronic total occlusions that have failed standard crossing techniques: results of the FAST-CTOs (Facilitated Antegrade Steering Technique in Chronic Total Occlusions) trial. JACC Cardiovasc Interv. 2012; 5(4): 393-401, doi: 10.1016/j.jcin.2012.01.014, indexed in Pubmed: 22516395.

22. Christopoulos G, Menon R, Karmpaliotis D, et al. Application of the "hybrid approach" to chronic total occlusions in a contemporary multicenter us registry. J Am Coll Cardiol. 2015; 65(10): A1796, doi: 10.1016/s0735-1097(15)61796-x. 